\title{
Measurement of small antenna reflector losses for radiometer calibration budget
}

\section{Skou, Niels}

Published in:

I E E E Transactions on Geoscience and Remote Sensing

Link to article, DOI:

$10.1109 / 36.602538$

Publication date:

1997

Document Version

Publisher's PDF, also known as Version of record

Link back to DTU Orbit

Citation (APA):

Skou, N. (1997). Measurement of small antenna reflector losses for radiometer calibration budget. I E E E Transactions on Geoscience and Remote Sensing, 35(4), 967-971. https://doi.org/10.1109/36.602538

\section{General rights}

Copyright and moral rights for the publications made accessible in the public portal are retained by the authors and/or other copyright owners and it is a condition of accessing publications that users recognise and abide by the legal requirements associated with these rights.

- Users may download and print one copy of any publication from the public portal for the purpose of private study or research.

- You may not further distribute the material or use it for any profit-making activity or commercial gain

- You may freely distribute the URL identifying the publication in the public portal

If you believe that this document breaches copyright please contact us providing details, and we will remove access to the work immediately and investigate your claim. 


\section{Communications}

\section{Measurement of Small Antenna Reflector Losses for Radiometer Calibration Budget}

Niels Skou

\begin{abstract}
Antenna reflector losses play an important role in the calibration budget for a microwave radiometer. If the losses are small, they are difficult to measure by traditional means. However, they can be assessed directly by radiometric means using the sky brightness temperature as incident radiation. This paper describes how such measurements are carried out as well as a suitable experimental setup. The main reflector of the European Space Agency's MIMR system is used to demonstrate the principle.
\end{abstract}

Index Terms-Antenna, radiometer, reflector loss.

\section{INTRODUCTION}

The fundamental pre-launch calibration of spaceborne radiometer systems is normally carried out with the instrument inside a thermal vacuum chamber which also includes a calibration target viewed by the radiometer's antenna system. The calibration target is very accurately temperature controlled and is able to provide brightness temperatures over a wide range, typically $77 \mathrm{~K}$ to $300 \mathrm{~K}$. Such a target is difficult and expensive to develop-especially if it has to be large in order to serve a large aperture system. Also, a thermal vacuum chamber for a large antenna is not a cheap nor trivial installation. For these reasons, it may be necessary to dismount the antenna reflector and carry out the calibration with the feed horns viewing the calibration target. Thus reflector losses must be accurately assessed so that they can be compensated for in the data analysis.

The reflection coefficient for a specimen of the reflector material can be measured with a network analyzer. However, if the reflection coefficient is close to one, which it will be for a decent reflector, it turns out to be difficult if not impossible to achieve the necessary measurement accuracy by this method. On the other hand it is really the emissivity of the reflector material that is needed for the radiometric calibration correction, and this can be measured directly and accurately down to very small values by radiometric means. The work to be described in the following has been inspired by work carried out previously by Carver [1] and Harrington [2].

\section{REFLECTOR LOSSES AND CALIBRATION BUdGET}

The reflector has a loss that modifies the measured brightness temperature. Basically we have the situation illustrated in Fig. 1.

The outgoing brightness temperature is found as:

$$
T_{B, o}=T_{B, i} \cdot(1-\varepsilon)+T_{R} \cdot \varepsilon
$$

where $T_{B, i}$ is the incoming brightness temperature, $T_{R}$ the physical temperature of the reflector, and $\varepsilon$ the emissivity. The reflectivity is equal to $1-\varepsilon$ in this case assuming no transmission takes

Manuscript received August 27, 1996; revised February 24, 1997. This work was supported by a contract with the European Space Agency through Alenia Spazio.

The author is with the Danish Center for Remote Sensing, Department of Electromagnetic Systems, Technical University of Denmark, DK-2800 Lyngby, Denmark (e-mail: ns@emi.dtu.dk).

Publisher Item Identifier S 0196-2892(97)04485-9.

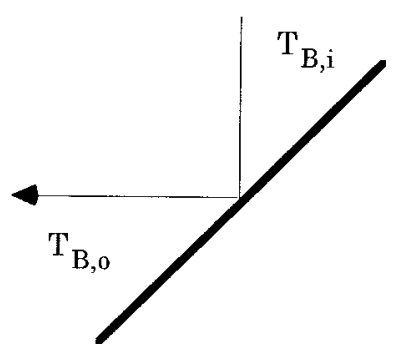

Fig. 1. Reflected brightness temperature.

place through the reflector. As an example, consider a situation in space where the sky temperature of $3 \mathrm{~K}$ is reflected in a reflector having an emissivity of 0.01 and a physical temperature of 300 $\mathrm{K}: T_{B, o}=2.97+3.0=5.97 \mathrm{~K}$. The brightness temperature is modified by some $3 \mathrm{~K}$, and, in general, the emissivity and the reflector temperature must be measured and a correction applied. Note that an emissivity of 0.01 corresponds to a loss of $0.04 \mathrm{~dB}$ which is a pretty low figure, difficult to measure accurately on a network analyzer.

Assume a radiometer system for which the absolute calibration budget includes $0.3 \mathrm{~K}$ uncertainty in the determination of signal loss due to reflector loss. For small emissivities, and the typical values for incoming brightness temperature and reflector temperature stated above, (1) shows that the brightness temperature is modified by reflection according to:

$$
\Delta T \cong \varepsilon \cdot T_{R}
$$

so the required uncertainty of $0.3 \mathrm{~K}$ in $\Delta T$ dictates a required knowledge of $\varepsilon$ to the 0.001 level (assuming good knowledge of the physical temperature $T_{R}$ ).

Preliminary network analyzer measurements of specimens of the MIMR reflector indicated an emissivity lower that 0.01 , warranting the exercises presented in the following.

\section{Measurement Setup}

The reflector to be measured is placed in a large metal bucket together with a radiometer and a suitable horn antenna. The device under test reflects the clear sky brightness temperature into the radiometer horn. The bucket diverts antenna sidelobes and spillover towards clear sky.

Fig. 2 shows how the reflector emissivity is measured in a threestep process using the sky temperature as radiation source.

First the sky temperature, $T_{B, S}$, is measured using a lossless plate (in this case a silver plated aluminum plate):

$$
T_{B, 1}=n \cdot T_{B, S}+(1-n) T_{B, S}=T_{B, S}
$$

where $n$ is the beam filling factor of the plate. $n$ is assumed close to one so that the major part of the energy emanent onto the antenna horn is sky temperature reflected in the lossless plate. The reflector and the bucket are assumed low loss, so that the sky temperature, reflected in these, weighted by $(1-n)$ and received by the horn antenna, is still a small term (a term that is balanced out in the following measurements anyway). Step (a) can be regarded as an inter-calibration of the radiometer and the sky temperature. 


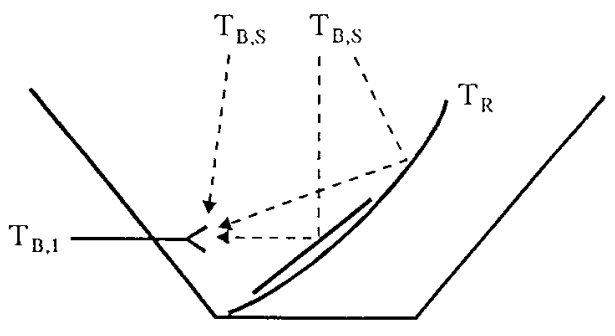

(a)

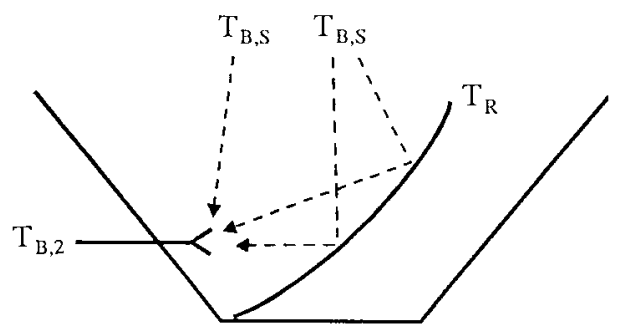

(b)

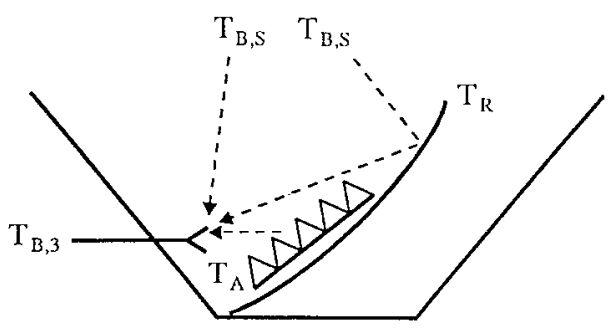

(c)

Fig. 2. Measurement schedule.

Secondly, the reflector alone is measured:

$$
T_{B, 2}=n\left(T_{R} \cdot \varepsilon+T_{B, S}(1-\varepsilon)\right)+(1-n) T_{B, S}
$$

where $T_{R}$ is the temperature of the reflector and $\varepsilon$ is its emissivity. It is now clear that this procedure is a substitution measurement comparing the lossless plate with an equal sized and shaped centre part of the reflector. If the reflector is very good (low loss) the difference between $T_{B, 1}$ and $T_{B, 2}$ is small.

Finally, in the third step (c) an absorber having the same physical size as the lossless plate is measured:

$$
T_{B, 3}=n \cdot T_{B, A}+(1-n) \cdot T_{B, S}
$$

where $T_{B, A}$ is the physical (hence also the brightness) temperature of the absorber.

Solving (3)-(5), we find

and

$$
n=\frac{T_{B, 3}-T_{B, 1}}{T_{B, A}-T_{B, 1}}
$$

$$
\varepsilon=\frac{T_{B, 2}-T_{B, 1}}{n\left(T_{R}-T_{B, 1}\right)} .
$$

It is clear that $T_{B, S}$ has to be constant throughout the experiment. So, only clear days without clouds can be used. Also the radiometer must be stable throughout an experiment. If the reflector is very good, the difference $T_{B, 2}-T_{B, 1}$ (which is the dominating factor in determining the emissivity) become small and the stability requirement becomes very stringent. During the experiments to be discussed later, the crucial steps, (a) and (b), were performed several times alternating between them. The time between successive measurements was kept
TABLE I

EMISSIVITY OF SOME CONDUCTORS

\begin{tabular}{l|c|l|c}
\hline & Conductivity & \multicolumn{2}{|c}{ Emissivity } \\
& $\sigma(\mathrm{s} / \mathrm{m})$ & $17 \mathrm{GHz}$ & $34 \mathrm{GHz}$ \\
\hline Silver & $6.17 \times 10^{7}$ & 0.00035 & 0.00049 \\
Copper & $5.80 \times 10^{7}$ & 0.00036 & 0.00051 \\
Aluminium & $3.72 \times 10^{7}$ & 0.00045 & 0.00064 \\
Brass & $1.57 \times 10^{7}$ & 0.00069 & 0.00098 \\
Iron & $1.12 \times 10^{7}$ & $0.00082 *$ & $0.0012 *$ \\
\hline
\end{tabular}

* neglecting magnetic properties at frequency in question.

to two minutes. Thus the time difference (important concerning effects of nonperfect stability) is short and measurements of the same situation are repeated enabling drifts to be checked.

The aluminum lined, plywood bucket has an opening of $3 \times 3$ $\mathrm{m}$ and a height of $1.2 \mathrm{~m}$. The MIMR reflector has a diameter of roughly $1.5 \mathrm{~m}$. The lossless plate is $0.5 \times 0.5 \mathrm{~m}$. Three noise-injection radiometers $(5,17$, and $34 \mathrm{GHz})$ were used for the measurements. They all exhibit equal sensitivities of $0.1 \mathrm{~K}$ for an integration time of $1 \mathrm{sec}$. as used during the experiments.

The antenna horns used for the experiments are rectangular standard gain horns. The aperture sizes are $6.9 \times 5.6 \mathrm{~cm}$ at $34 \mathrm{GHz}$, $15 \times 12.5 \mathrm{~cm}$ at $17 \mathrm{GHz}$, and $22 \times 18 \mathrm{~cm}$ at $5 \mathrm{GHz}$. The $34-\mathrm{GHz}$ and the $17-\mathrm{GHz}$ horns have a beamwidth of about $10^{\circ}$. The $5 \mathrm{GHz}$ beamwidth is about twice of that, but the lossless plate is very close to the horn where the beam is of roughly the same size as the aperture.

\section{THEORETICAL EMISSIVITY}

So far, the concept of a so-called lossless plate has been assumed. Since the MIMR reflector is expected to exhibit very low losses, it is necessary to consider if the silver plated aluminum plate can be assumed lossless.

With the notation $R_{s}=$ surface resistivity, and $\eta=$ impedance of intrinsic medium, the power reflectivity $(\rho)$ for a good conductor is [3]

$$
\rho=1-\frac{4 R_{s}}{\eta}
$$

$\eta=377 \Omega$ when the intrinsic medium is air as in this case. $\rho=1-\varepsilon$ for the case of no transmission, which we can assume with good confidence, considering the relatively thick metal plates.

The surface resistivity, $R_{s}=\sqrt{\frac{\pi f \mu}{\sigma}}$, or for nonmagnetic material

$$
R_{s}=\sqrt{\frac{\pi \cdot f \cdot \mu_{0}}{\sigma}}=2 \pi \cdot \sqrt{\frac{f}{\sigma \cdot 10^{7}}},
$$

where $\sigma$ is the conductivity, and $f$ the frequency. Hence we find

$$
\varepsilon=\frac{1}{15} \sqrt{\frac{f}{\sigma \cdot 10^{7}}} .
$$

Inserting the conductivity for typical metals, the values shown in Table I are found. The difference between silver and copper is minute but aluminum is also very close and the bucket can be regarded as almost lossless (as earlier discussed and assumed).

If we want to measure emissivities in the 0.01 range, then the silver plate can be considered lossless. However, if emissivity measurements in the range around 0.001 are desired, then the silver plate is no longer "lossless" and corrections are needed if quantative measurements are to be carried out. 
Brightness temperature (K)

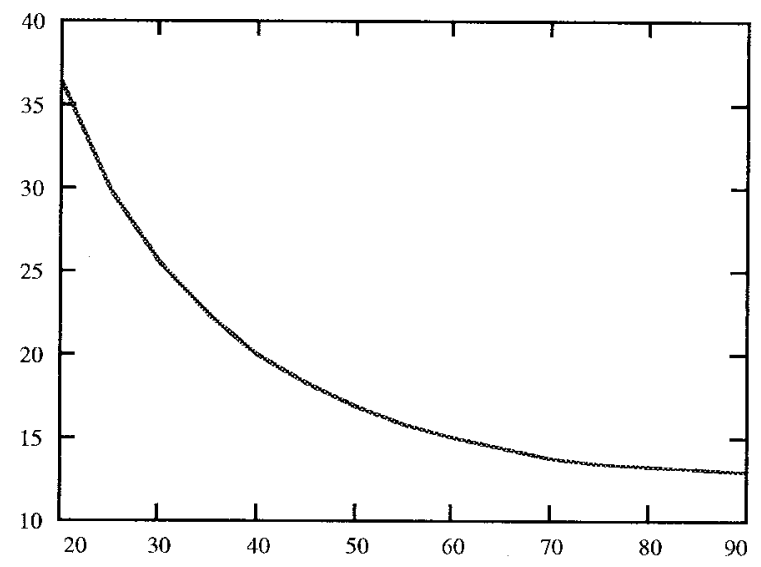

Elevation angle $\left(^{\circ}\right)$

Fig. 3. Brightness temperature of the sky at $17 \mathrm{GHz}$ as function of elevation angle, typical summer day.

\section{SKy BRIGHTNESS TEMPERATURE}

The sky brightness temperature is, in principle, measured in step (a) by means of the radiometer. This would require a well calibrated radiometer, and the best way to calibrate a radiometer to facilitate accurate measurements of very low temperatures is to use the sky as calibration target. Hence, in practise, step (a) is the calibration of the radiometer assuming knowledge of the sky temperature. The sky temperature need not be known with any great accuracy (note how it enters the formulas in Section III) but it must be stable during a measurement (all steps)! Hence, only clear days can be used. Typical values of some $5 \mathrm{~K}$ at $5 \mathrm{GHz}, 8-14 \mathrm{~K}$ at $17 \mathrm{GHz}$, and $12-18 \mathrm{~K}$ at $34 \mathrm{GHz}$ (depending on humidity) have been used.

So far, only the zenith sky temperature has been considered. However, it is of interest to assess how the sky temperature changes as a function of angle away from the zenith direction.

It can be shown [4] that in a horizontally stratified atmosphere the dependence of sky temperature on elevation angle is given by the equation

$$
T_{s}(\theta)=T_{o}\left(1-\beta^{\operatorname{cosec} \theta}\right)
$$

where $\theta$ is the elevation, $T_{o}$ the average temperature of the atmosphere (here taken as $278 \mathrm{~K}$ ), and $\beta$ is a constant fitted to give the correct zenith temperature at the frequency in question. Fig. 3 shows the $17-\mathrm{GHz}$ brightness temperature as a function of elevation angle on a typical summer day. A few discrete values are: $13.0 \mathrm{~K}$ for zenith look, $13.2 \mathrm{~K}$ for $80^{\circ}$ elevation, and $13.8 \mathrm{~K}$ for $70^{\circ}$ elevation. It is seen that zenith looking in itself is not very critical.

\section{Emissivity Resolution, Measurements on Metal Plates}

The potential "emissivity resolution", i.e., the lower limit of what can be measured with the present setup, can be estimated using (7)

$$
\Delta \varepsilon=\frac{\sqrt{2} \cdot \delta T}{n\left(T_{R}-T_{B, 1}\right)}
$$

where $\delta T$ is the radiometric sensitivity. Inserting typical figures $n=0.98, T_{R}=300 \mathrm{~K}, T_{B, 1}=10 \mathrm{~K}$, and $\delta T=0.1 \mathrm{~K}$, we find $\Delta \varepsilon=0.0005$. This is adequate considering the 0.001 requirement stated in Section II.

It is of interest to assess the emissivity resolution experimentally. To this end, different metal plates were measured against the lossless

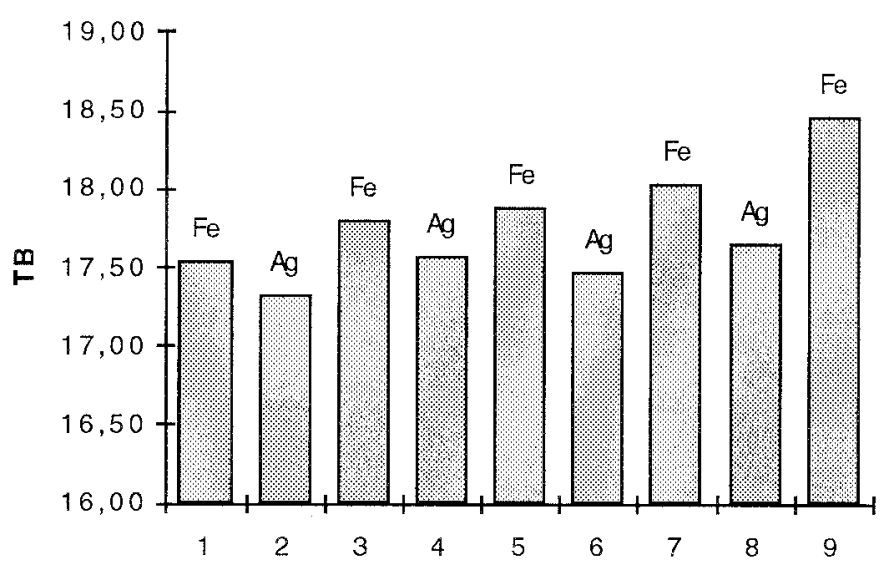

Fig. 4. $34 \mathrm{GHz}$ measurement of iron and silver plates.

plate in the bucket. The procedure was like that illustrated in Fig. 2, but in place of the reflector a jig was mounted to the bottom of the bucket. This jig holds the metal plates (and the absorber) in front of the antenna horn. Just as in the normal measurement procedure, the test plate and the lossless plate were measured several times alternating between them and with two minutes between measurements. The absorber was also measured to determine the beam filling factor the usual way. The test plates are, of course, exactly the same size as the "lossless" plate.

Fig. 4 presents results from a preparatory exercise measuring the difference between an iron plate and the lossless plate. The magnetic properties of the iron plate are not known so the actual value of the emissivity is not known. It is evident from looking at the bar diagram in Fig. 4 that the iron plate has an emissivity large enough to be measurable by the method employed. The reflected brightness temperatures are, in the iron case, systematically larger than in the lossless case. By averaging, we find for iron: $T_{B, 2}=17.94 \mathrm{~K}$, for the lossless case: $T_{B, 1}=17.50 \mathrm{~K}$. Inserting in formulas (6) and (7), we find: $n=0.9814$ and $\varepsilon=0.0017$. The beam filling factor is good. The emissivity value and the systematic behavior of the bar diagram lead us to state that the method can measure emissivity values somewhat smaller than 0.0017 .

Lack of stability is evident. Note how the brightness temperature, especially in the iron case, drift with time. Drift problems are also seen in other figures to follow, and certainly experienced during the experiments. They are caused by instabilities in the atmosphere and in the radiometers, and drive the requirement for short intervals between steps in the measurement procedure. Two minutes was found to be the shortest possible for the present setup.

In an attempt to find the limiting value of emissivity that can be measured, a brass plate was used in the following experiments. Fig. 5 reports a $17-\mathrm{GHz}$ measurement and it is noted that good stability prevails but there is no systematic pattern in the bar chart. The calculated emissivity is as low as $\varepsilon=-0.0002$, also indicating that we cannot see the difference between brass and silver. Hence, we cannot measure the emissivity of brass at $17 \mathrm{GHz}(0.00069$ theoretically).

The next experiment, see Fig. 6, concerns the measurement of brass at $34 \mathrm{GHz}$. At this frequency the theoretical emissivity is $\varepsilon=0.00098$. A careful inspection of the bar chart shows that in all cases except measurement "4," going from brass to silver results in a lower brightness temperature while going from silver to brass results in a higher brightness temperature. Hence, there is a systematic and reasonable pattern in the bars and we can feel confident that we can perform an emissivity assessment. By proper averaging and using the 


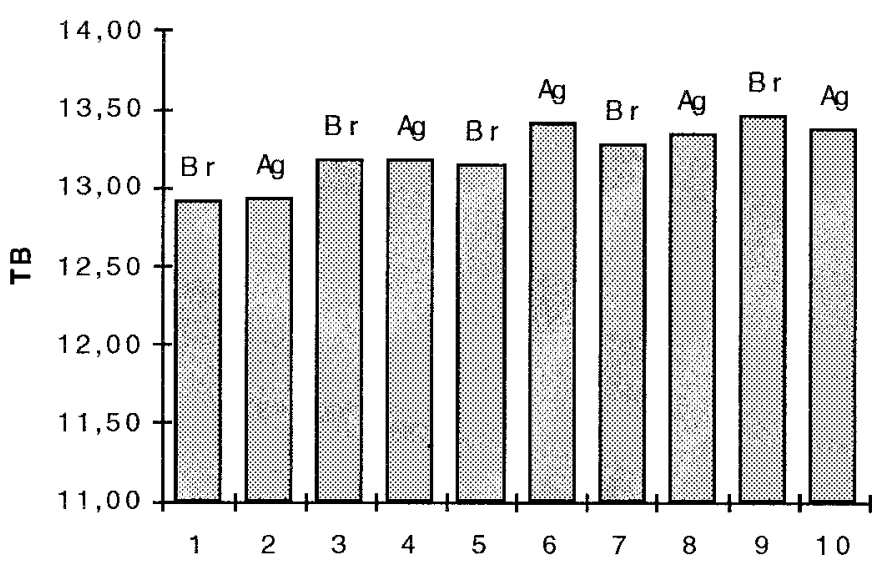

Fig. 5. A $17-\mathrm{GHz}$ measurement of brass and silver plates.

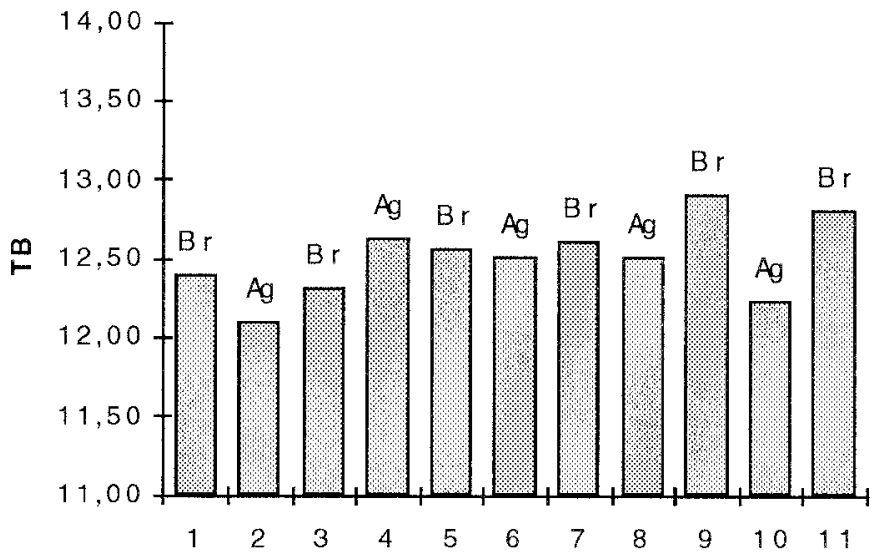

Fig. 6. A 34-GHz measurement of brass and silver plates.

usual formula we find $\varepsilon=0.0008$ which is in fair agreement with the theoretical value, bearing in mind that we have not corrected for losses in the so-called "lossless" plate.

Hence, it is evident that we can measure an emissivity of about 0.001 . However, the results from similar $34-\mathrm{GHz}$ experiments not reported here show that, in some cases, we can see the difference between the lossless plate and the brass plate, in others not. In no case can a difference be detected at $17 \mathrm{GHz}$. (Note that since the different radiometers exhibit similar sensitivities, we can directly compare results obtained at different frequencies.) This indicates that even 0.001 is marginal and we can certainly not measure smaller values. The reason for this lower limit is measurement noise in form of a combination of radiometer instabilities, atmosphere instabilities, and radiometric sensitivity. The radiometric sensitivity is, as we have seen earlier, adequate, so the limiting factors are the instabilities.

We conclude that the lower limit for emissivity measurable by the present setup is 0.001 , which fits our requirements, and we note that this is considerably better than what is possible with a network analyzer.

\section{MIMR REFLECTOR MEASUREMENTS}

In the following, two examples of measurements on the MIMR reflector itself shall be presented. Fig. 7 shows 34-GHz results, and (a) and (b) refer to the measurement steps (see Fig. 2). Stability is good and by proper averaging we find:

$T_{B, 1}=13.02 \mathrm{~K}, T_{B, 2}=13.07 \mathrm{~K}, T_{B, A}=T_{R}=273$ $\mathrm{K}, T_{B, 3}=252.11 \mathrm{~K}$, and using the usual formulas (6) and (7) $n=0.9196, \varepsilon=0.0002$.

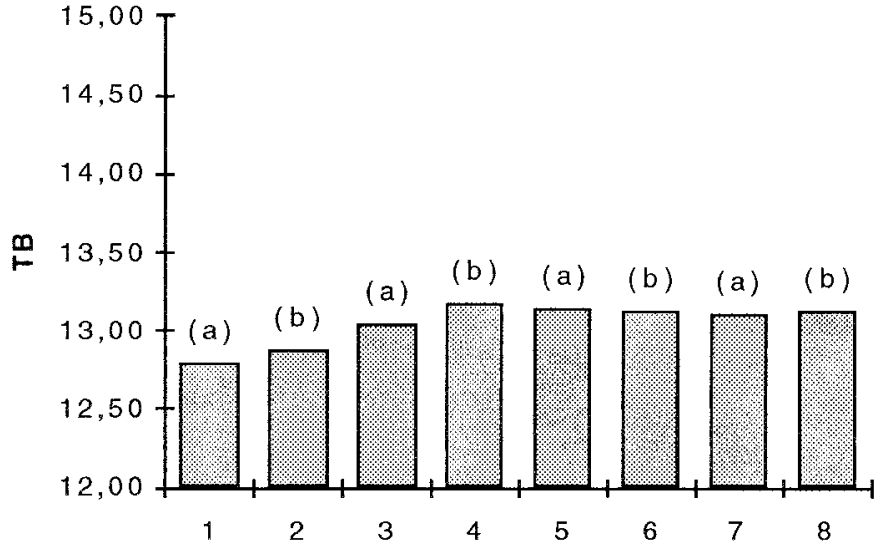

Fig. 7. A 34-GHz measurement of the MIMR reflector.

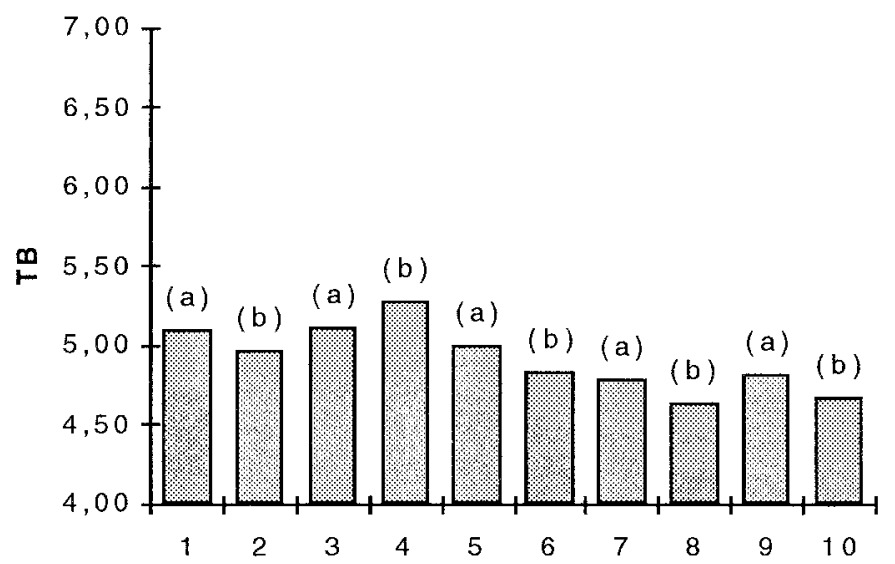

Fig. 8. A 5-GHz measurement of the MIMR reflector.

The beam filling factor is good, but we cannot measure any difference between the reflector and the lossless plate. This is indicated by the low emissivity value, and also evident from the bar diagram in Fig. 7.

Several experiments were carried out, spread out over the period from August to December, covering physical temperatures from 27 ${ }^{\circ} \mathrm{C}$ to $-4{ }^{\circ} \mathrm{C}$. In no case could any statistically significant difference be observed. This was also the case at $17 \mathrm{GHz}$.

The $5-\mathrm{GHz}$ measurements are of special interest. The MIMR reflector is made of carbon fiber with a very thin $(1 \mu \mathrm{m})$ metallization as reflecting surface. One could be concerned about the performance at the low frequency with its larger skin depth.

Fig. 8 shows an example of the $5-\mathrm{GHz}$ measurements. We find $n=0.8824$ and $\varepsilon=-0.0003$, and the situation is as for the other frequencies: we cannot measure the difference between the MIMR reflector and the lossless plate.

\section{Discussion OF THE MEASUREMENTS}

The different emissivity measurements on the MIMR reflector are summarized in Table II.

The first $17-\mathrm{GHz}$ and $34-\mathrm{GHz}$ measurements (22/8) used quite long distance between the horn and the reflector $(1 \mathrm{~m})$ leading to a poor beam filling factor around $75 \%$, and to awkward negative emissivity values, indicating that the MIMR reflector is better that the lossless plate which is, of course, nonsense. The rest of the measurements used shorter distances leading to beam filling factors in the $90 \%$ to $95 \%$ range. 
TABLE II

SumMary of EMISSIVITy MeasuremENTS

\begin{tabular}{l|c|c|c|c|c}
\hline Date & $22 / 8$ & $23 / 8$ & $7 / 11$ & $13 / 11$ & $20 / 12$ \\
Temperature & $27^{\circ}$ & $27^{\circ}$ & $0^{\circ}$ & $2^{\circ}$ & $-4^{\circ}$ \\
\hline $34 \mathrm{GHz}$ & -0.0014 & -0.0001 & 0.0002 & & -0.0003 \\
$17 \mathrm{GHz}$ & -0.0029 & -0.0001 & -0.0005 & & 0.0003 \\
$5 \mathrm{GHz}$ & & & -0.0017 & -0.0003 & -0.0001 \\
\hline
\end{tabular}

The first $5 \mathrm{GHz}$ measurement (7/11) also suffered from a long distance hence inadequate beam filling. This was corrected for the later measurements.

The table shows that as soon as an adequate beam filling factor (some 90\%) is achieved, we consistently get very small values indicating that we cannot see the difference between the MIMR reflector and the lossless plate. The fact that some of these very small values are negative is not significant, but merely reflects the statistical nature of radiometric measurements.

What is the reason for the awkward, nonphysical, behavior where inadequate beam filling leads to negative emissivities? Two mechanisms have been considered.

1) Focusing effect: In the first cases (22/8 measurements), the distance between the reflector and the antenna horns was comparable to the focal length of the reflector. Thus the $10^{\circ}$ beam of the antenna horn tended to be focused in the zenith direction when measuring the reflector but not when measuring the lossless plate. Thus, in the latter case a comparatively larger portion of the incident radiation emanated from the warmer sky off zenith (see Fig. 3) which would bias the measurements in the observed direction.

2) Edge effects: When the beam filling factor is inadequate, the edges of the lossless plate contribute significantly to the received radiation by reflecting portions of the sky temperature way off zenith into the antenna horn. The bias from these contributions can only increase the measured temperature, since the zenith brightness temperature is the lowest possible, and this could explain the observed problems.

Measurements at the two higher frequencies show that when the beam filling factor is adequate (around 90\%), its absolute value does not matter. Experiments not reported here, for which the distance was varied to yield beam filling factors between $90 \%$ and $95 \%$, indicated no measurable differences.

The measurement of the physical temperature of the reflector and the absorber shall now be described. Recalling (2) we find that for $\varepsilon=0.001$ and a reflector temperature of $300 \mathrm{~K}$, the reflected brightness temperature is modified by $\Delta T=0.30 \mathrm{~K}$. If we change the physical temperature by $10^{\circ}$ to $290 \mathrm{~K}$, we find $\Delta T=0.29 \mathrm{~K}$. This means that an error in the knowledge of the physical temperature of $10{ }^{\circ} \mathrm{C}$ results in an error on the reflected sky brightness temperature of only $0.01 \mathrm{~K}$, assuming a good reflector with $\varepsilon=0.001$. We have a good reflector and it is perfectly adequate to assess its physical temperature by just measuring the ambient temperature. The absorber temperature is also assessed the same way. This may give some small errors in the determination of the beam filling factor, which in turn leads to small errors in the measured emissivities. These are approaching zero anyway and are below our measurement capabilities. Some things become simple when the reflector to be measured is as good as the MIMR reflector.

\section{CONCLUSION}

- The experiments with different metal plates indicate that the present measurement setup can measure emissivities down to the 0.001 level. This is considerably better than what is possible with a network analyzer.

- The experiments with the MIMR reflector show that in the frequency range 5-34 GHz we cannot see any difference between the reflector and the lossless plate. It is believed that the emissivity of the reflector, which is manufactured in carbon fiber and coated with a thin layer of aluminum, approaches that of an aluminum plate (emissivity in the range of 0.00024-0.00064). This cannot, however, be proven by the present measurements due to the $\varepsilon=0.001$ limit stated above. But we can conclude that in the frequency range $5-34 \mathrm{GHz}$ the emissivity of the MIMR reflector is below 0.001. This would not have been possible based on network analyzer measurements.

- Measurements have been carried out in the temperature range $+27{ }^{\circ} \mathrm{C}$ to $-4{ }^{\circ} \mathrm{C}$. No dependence on temperature is found.

- The sky brightness temperature reflected in a reflector having an emissivity of 0.001 and a physical temperature of $300 \mathrm{~K}$ is only modified by $0.3 \mathrm{~K}$. The somewhat higher brightness temperatures from Earth targets are modified correspondingly less (some $0.2-\mathrm{K}$ worst case). This means that correction for MIMR main reflector losses are hardly warranted. $0.2 \mathrm{~K}$ must be included in the calibration budget.

- If better values for the emissivity of a reflector are needed, a possible approach is to adopt a slightly different measurement concept: a specimen of the reflector material having the shape and size of the lossless plate must be manufactured. The specimen and the lossless plate would again be measured alternatively in the metal bucket. It is possible to make a mechanism so that the specimen and the lossless plate could be interchanged in seconds-speed is a major factor considering instrument and atmosphere instabilities. At the same time, the measurement geometry would be consistent and edge effects would be cancelled. This way very accurate measurements would be possible.

\section{ACKNOWLEDGMENT}

R. Bordi and P. Spera of Alenia have contributed to the work through fruitful discussions.

\section{REFERENCES}

[1] K. R. Carver, "Antenna and radome loss measurements for MFMR and PIMS," Rep., Physical Sci. Lab., New Mexico State Univ., Las Cruces, 1975.

[2] R. F. Harrington and H.-J. C. Blume, "Determination of electromagnetic properties of mesh material using advanced radiometer techniques," NASA Langley Res. Ctr., in NASA Conf. Publ. 2368: Large Space Antenna Systems Technology, 1984.

[3] S. Ramo, J. R. Whinnery, and T. van Duzer, Fields and Waves in Communication Electronics. New York: Wiley, 1994.

[4] A. D. Sayers, "Radiometric sky temperature measurements at $35 \mathrm{GHz}$ and 89 GHz," in Inst. Elec. Eng. Proc., June 1986, vol. 133, pt. H, no. 3, pp. 233-237. 